\title{
THE NIELSEN REALIZATION PROBLEM
}

\author{
BY STEVEN P. KERCKHOFF 1
}

If $M_{g}$ is a closed, oriented 2-manifold of genus $g \geqslant 2$, then it admits many hyperbolic metrics (metrics of constant curvature -1 ). In special cases such a metric possesses a nontrivial group of symmetries, of isometries to itself. The group of isometries of a closed hyperbolic manifold is always finite and the only isometry isotopic to the identity is the identity itself. Thus a group of symmetries of a hyperbolic surface determines an isomorphic finite subgroup of the group of isotopy classes of diffeomorphisms of $M_{g}$. The purpose of this paper is to announce a positive solution to the Nielsen Realization Problem that the converse is true, i.e.; the

THEOREM 1. Every finite subgroup of $\pi_{0}$ Diff $M_{g}$ can be realized as a group of isometries of some hyperbolic structure on $M_{g}$.

For $g \geqslant 2$ the map Diff $M_{g} \rightarrow \pi_{0}$ Diff $M_{g}$ is a homotopy equivalence, but it is unknown whether or not $\pi_{0}$ Diff $M_{g}$ can be lifted back into Diff $M_{g}$ as a subgroup. Theorem 1 solves the lifting problem for finite subgroups of $\pi_{0}$ Diff $M_{g}$.

We will call $\pi_{0}$ Diff $M_{g}$ the modular group $2\left(\operatorname{Mod}_{g}\right)$ since it is the natural generalization of the classical modular group for $g=1$. Another common name is the mapping class group. 1 It is naturally isomorphic to the group of outer automorphisms of $\pi_{1} M_{g}$. $\operatorname{Mod}_{g}$ acts on the Teichmüller space $\left(T_{g}\right)$ of hyperbolic metrics on $M_{g}$ (where two are considered equivalent if there is an isometry, isotopic to the identity, between them). $T_{g}$ is homeomorphic to a $(6 g-6)$-dimensional ball and the action of $\operatorname{Mod}_{g}$ on $T_{g}$ is properly discontinuous and, except for an element of order two in genus two, faithful. Theorem 1 is equivalent to

THEOREM 2. Every finite subgroup of $\operatorname{Mod}_{g}$ acting on $T_{g}$ has a fixed point.

For $g=1, T_{g}$ (for flat metrics of area 1 ) is the upper half plane, $\operatorname{Mod}_{g} \approx$ $G L(2, \mathbf{Z})$ acts by linear fractional transformations, and Theorem 2 is classical. In the higher genus case, Theorems 1 and 2 were known for $G$ cyclic (Nielsen [4]), solvable (Fenchel [1]), and in many other special cases (see Zieschang [6]). Kravetz [2] gave a proof of Theorem 2, but it was based on the false belief that $T_{g}$, equipped with Teichmüller's metric, has negative curvature (see Masur [3]).

Received by the editors November 21, 1979.

AMS (MOS) subject classifications (1970). Primary 55A99, 20H10, 32G15; Secondary $30 \mathrm{~A} 58,53 \mathrm{~A} 35,57 \mathrm{D} 50$.

1 The author wishes to thank the AMS and NSF for support and the Institute for Advanced Study for space during the period when this work was done.

2 We make no restriction to the orientation-preserving case, however. 
Let $S$ denote the set of isotopy classes of simple closed curves on $M_{g}$. If $\phi \in S$ then on any hyperbolic surface there is a unique geodesic isotopic to $\phi$. Let $l_{\phi}: T_{g} \rightarrow \mathbf{R}_{+}$denote the function which assigns to the point $x \in T_{g}$ the geodesic length of $\phi$ w.r.t. the metric $x$. When $\phi$ is a finite set of $\phi_{i} \in S$, let $l_{\phi}$ denote the sum $\Sigma_{\phi_{i} \in \phi} l_{\phi_{i}}$. $\operatorname{Mod}_{g}$ acts on $S$ so let $G \phi$ denote the orbit of $\phi$ under the finite group $G \subset \operatorname{Mod}_{g}$. Then the function $l_{G \phi}$ is $G$-invariant. The idea of the proof of Theorem 2 is to show that, if the set $G \phi$ is large enough, then $l_{G \phi}$ realizes a unique minimum in $T_{g}$. Since $l_{G \phi}$ is $G$-invariant, this minimum is a fixed point for the group $G$.

Definition. A collection $\phi=\left\{\phi_{i}\right\} \in S$ is said to fill up $M_{g}$ if, when the $\phi_{i}$ are isotoped to have a minimal number of pairwise intersections, then every component of $M_{g}-\bigcup \phi_{i}$ is topologically a disk. Equivalently, every $\tau \in S$ must intersect at least one of the $\phi_{i}$ when they are in this minimal position.

Proposition 1. If $\phi=\left\{\phi_{i}\right\} \in S$ fills up $M_{g}$, then $l_{\phi}: T_{g} \rightarrow \mathbf{R}_{+}$has $a$ unique minimum in $T_{g}$.

In order to prove Proposition 1 we study the behavior of the function $l_{\phi}$ along certain horocycle-like paths in $T_{g}$. The prototype for such a path is the 1-parameter family of surfaces gotten by choosing a simple geodesic on a hyperbolic surface, cutting along it, and glueing it back together with a left-hand twist of distance $t$. For each $t \in \mathbf{R}_{+}$the result is a new hyperbolic structure on $M_{g}$.

In general one can define a 1-parameter family of deformations by sheering along a geodesic lamination with transverse measure $\mu$ (g.1. $\mu$. for short) (see Thurston [5]). These deformations were invented by Thurston who named them left earthquakes. A path in $T_{g}$ determined by earthquaking along the g.1. $\mu . v$ will be denoted by $h_{v}$. The key step in proving Proposition 1 is

Proposition 2. The function $l_{\phi}$ is convex along $h_{v}$. It is strictly convex iff the intersection number of $\phi$ with $v$ is nonzero.

Finally we need to know that every two points in $T_{g}$ are connected by some $h_{v}$. This fact is due to Thurston (unpublished) and will appear in later chapters of $[5]$.

Theorem 3 (Thurston). For any $x, y \in T_{g}$ there is a unique left earthquake taking $x$ to $y$.

When $\phi$ fills up $M_{g}$, it is not hard to see that $l_{\phi}$ is a proper function. Using this fact, together with Theorem 3 and Proposition 2, it is easy to prove Proposition 1 and hence Theorem 2.

We remark that the methods used here work for a general Teichmüller space, when the hyperbolic manifold is allowed to have geodesic boundary or cone-like singularities and that the theorems were stated for closed surfaces only 
to avoid the details involved in defining these structures and their modular groups. The nonorientable case can be handled by passing to the oriented double cover.

Finally, the Nielsen Problem is equivalent to describing when a finite extension of a surface group is again a surface group (in the generalized sense of a surface with cone-like singularities, etc.) and provides some new information about the homotopy type of 3-manifolds which have finite covering spaces which are Seifert manifolds. These subjects will be deferred to a format with more space.

\section{BIBLIOGRAPHY}

1. W. Fenchel, Estensioni di gruppi discontinui e transformazioni periodiche delle superficie, Rend. Accad. Naz. Lincei 5 (1948). 326-329.

2. S. Kravetz, On the geometry of Teichmüller spaces and the structure of their modular groups, Ann. Acad. Soc. Sci. Fenn. 278 (1959), 1-35.

3. H. Masur, On a class of geodesics in Teichmüller space, Ann. of Math. (2) 102 (1975), 205-221.

4. J. Nielsen, Abbildungklassen Endlicher Ordung, Acta Math. 75 (1943), 23-115.

5. W. Thurston, The geometry and topology of 3-manifolds (preprint).

6. H. Zieschang, Lecture Notes in Math. (to appear).

DEPARTMENT OF MATHEMATICS, UNIVERSITY OF CALIFORNIA, BERKELEY, CALIFORNIA 94720 